\title{
COVID-19 and related symptoms in patients under disulfiram for alcohol use disorder
}

\author{
Stefano Tamburin ${ }^{1}$ (1) Elisa Mantovani ${ }^{1}$ - Ernesto De Bernardis ${ }^{2} \cdot$ Donato Zipeto $^{1} \cdot$ Fabio Lugoboni $^{3} \cdot$ Gruppo \\ InterSERT di Collaborazione Scientifica (GICS)
}

Received: 25 November 2020 / Accepted: 4 January 2021 / Published online: 19 January 2021

(c) Società Italiana di Medicina Interna (SIMI) 2021

Keywords Alcohol related liver disease $\cdot$ Alcohol use disorder · COVID-19 · Disulfiram · SARS-CoV-2 · Treatment

Dear Editor,

COVID-19, a severe acute respiratory syndrome (SARS) due to a new SARS coronavirus (SARS-CoV-2) infection, caused a global pandemic with $>58$ million cases and nearly 1.4 million deaths so far.

There is no established treatment for SARS-CoV-2. Disulfiram, a hepatic aldehyde dehydrogenase inhibitor approved for alcohol aversion therapy, may inhibit the SARS coronavirus proteases [1], but clinical evidence on SARSCoV-2 is lacking [2].

We explored whether patients under disulfiram for alcohol use disorder (AUD) had reduced COVID-19 and related symptoms.

This is a multicenter observational telephone interview on patients aged $>18$ with AUD living in Northern Italy (Lombardy, Veneto, Emilia Romagna, Piedmont, and Liguria regions), where the first COVID-19 peak was more severe in spring 2020.

Patients were asked on laboratory-confirmed COVID19 (SARS-CoV-2 positivity by polymerase chain reaction, PCR; primary outcome), hospitalization related to

The members of Gruppo InterSERT di Collaborazione Scientifica (GICS) are listed in "Acknowledgements".

Stefano Tamburin

stefano.tamburin@univr.it

1 Department of Neurosciences, Biomedicine and Movement Sciences, University of Verona, Piazzale Scuro 10, 37134 Verona, Italy

2 Addiction Medicine Unit, Siracusa Province Health Agency, Lentini, Italy

3 Department of Medicine, Addiction Medicine Unit, Verona University Hospital, Verona, Italy
COVID-19, pneumonia, and symptoms compatible with COVID-19 (fever, dyspnea; secondary outcomes) [3] from February 21st to May 31st, 2020, demographic and clinical covariates, disulfiram treatment and dosage.

Fisher's exact test was applied to categorical variables. For continuous variables, Student's $t$ test and Mann-Whitney $U$ test were applied. Multivariate logistic regression backward models explored the effect of disulfiram and the covariates, which significantly differed between groups at baseline (i.e., age, liver disease, and opioid abuse), on symptoms compatible with COVID-19 (fever, dyspnea, either fever, or dyspnea; binary dependent variable). $P<0.05$ (twotailed) was the significance threshold.

We included 1297 patients (age 50.1 \pm 10.3 , range 21-79; 881 men, 418 women), of whom 752 under disulfiram (dosage $241.7 \pm 112.3$, median 200 , range $100-800 \mathrm{mg}$; disulfiram treatment duration $14.0 \pm 9.3$, median 10 , range 3-120 months) and 545 not taking disulfiram (control group). Among baseline demographic and clinical covariates, age, liver disease, and opioid abuse significantly differed between groups (Table 1).

Laboratory-confirmed COVID-19 $(N=10,0.77 \%)$, hospitalization due to confirmed COVID-19, and pneumonia were less, but not significantly, common in disulfiram group. Symptoms compatible with COVID-19 were significantly less common in disulfiram group (Table 1).

The multivariate logistic regression model showed significantly reduced risk of fever (unadjusted odds ratio, OR 0.39 [95\% CI 0.18-0.81]; adjusted OR: 0.37 [95\% CI $0.18-0.77$ ] $p=0.007$ ), dyspnea (unadjusted OR 0.50 [95\% CI 0.31-0.82]; adjusted OR 0.53 [95\% CI 0.32-0.85], $p=0.009$ ), either fever or dyspnea (unadjusted OR 0.44 [95\% CI 0.28-0.72]; adjusted OR 0.45 [95\% CI 0.29-0.70], $p<0.001)$ for disulfiram group, while the covariates that 
Table 1 Demographic and clinical characteristics of the participants and outcome measures

\begin{tabular}{llll}
\hline Variables & Disulfiram $(N=752)$ & Control $(N=545)$ & $p$ value \\
\hline Baseline-demographic & & & \\
Gender-M/W (\%) & $501 / 251(66.6 / 33.4 \%)$ & $380 / 165(69.7 / 30.3 \%)$ & 0.25 \\
Age $(\text { years) })^{\mathrm{a}}$ & $49.5 \pm 10.3,50$ & $50.8 \pm 10.3,51$ & $0.03^{*}$ \\
BMI $\left(\mathrm{Kg} / \mathrm{m}^{2}\right)^{\mathrm{a}}$ & $25.1 \pm 4.1,24.6$ & $25.0 \pm 4.1,24.5$ & 0.6 \\
Baseline—clinical一no. (\%) & & & \\
Diabetes & $28(3.7 \%)$ & $29(5.3 \%)$ & 0.17 \\
Hypertension & $108(14.4 \%)$ & $68(12.5 \%)$ & 0.37 \\
COPD & $18(2.4 \%)$ & $19(3.5 \%)$ & 0.31 \\
Liver disease & $43(5.7 \%)$ & $71(13.0 \%)$ & $<0.001^{*}$ \\
Heart disease & $25(3.3 \%)$ & $28(5.1 \%)$ & 0.12 \\
Smoke & $390(51.9 \%)$ & $266(48.8 \%)$ & 0.29 \\
Opioid abuse & $19(2.5 \%)$ & $28(5.1 \%)$ & $0.016^{*}$ \\
Outcome—no. (\%) & & & 0.75 \\
Laboratory-confirmed COVID-19 & $5(0.66 \%)$ & $5(0.92 \%)$ & 0.73 \\
Hospitalization due to confirmed COVID-19b & $4(0.53 \%)$ & $4(0.73 \%)$ & 0.14 \\
Pneumonia & $4(0.53 \%)$ & $8(1.47 \%)$ & \\
Symptoms compatible with COVID-19 & & & $0.006^{*}$ \\
Fever & $11(1.46 \%)$ & $22(4.04 \%)$ & $0.0012^{*}$ \\
Dyspnea & $30(3.99 \%)$ & $40(7.34 \%)$ & $<0.001^{*}$ \\
Either fever or dyspnea & $34(4.52 \%)$ & $52(9.54 \%)$ &
\end{tabular}

$B M I$ body mass index, $C O P D$ chronic obstructive pulmonary disease

${ }^{\mathrm{a}} \mathrm{Mean} \pm \mathrm{SD}$, median

${ }^{b}$ All patients who were hospitalized due to confirmed COVID-19 were also reported as laboratory-confirmed COVID-19 cases

*Significant between-group comparison differed at baseline (age, liver disease, opioid abuse) were not significant.

Among liver disease patients $(N=114)$, none in disulfiram group reported laboratory-confirmed COVID-19 or related hospitalization (control: $N=1$ ), pneumonia (control: $N=4$ ), and symptoms compatible with COVID-19 (control: fever, $N=6$; dyspnea, $N=4$ ), but the difference was not significant.

We found no significant difference for laboratory-confirmed COVID-19, related hospitalization, or pneumonia, but symptoms compatible with COVID-19 were significantly less common in disulfiram group. Multivariate model confirmed disulfiram to carry significantly reduced risk of symptoms compatible with COVID-19.

We found $0.77 \%$ of the cohort to have laboratory-confirmed COVID-19 positivity, in line with Northern Italy prevalence at the time of the study (i.e., around 0.9\%) [4], suggesting our patients to be representative of the general population. Our sample had slightly higher mean age and prevalence of men than the general population, but the risk of symptomatic SARS-CoV-2 infection is similar among adults, regardless of age [3].

The study failed the primary outcome, but laboratoryconfirmed COVID-19 percentage was smaller in disulfiram group. The reduced PCR availability during the first COVID19 peak might have contributed to this finding. In accordance with estimates of Italian population infected by SARSCoV-2 [5], symptoms compatible with COVID-19 were 10 times more common than laboratory-confirmed positivity in controls, suggesting this secondary outcome may represent a good proxy of COVID-19 cases [3]. Non-SARS-CoV-2 viral infections should not be a bias, being equally distributed between groups [3].

None of the liver disease patients under disulfiram had laboratory-confirmed COVID-19 and symptoms compatible with COVID-19, but the small size prevented a robust statistical analysis. These data might be of interest, because altered liver function tests were reported to be highly prevalent in COVID-19 patients and associated with worse outcome [6].

The study strengths are the large sample size, similar baseline characteristics, no influence of covariates that differed between groups. The limitations are the retrospective observational design, the small number of PCR-confirmed cases that might have resulted in missing mild/asymptomatic SARS-CoV-2 infections [3], and not having considered other symptoms (i.e., myalgia, arthralgia, fatigue, and headache) as compatible with COVID-19. 
Because of the discrepancy between patients with and without laboratory confirmed COVID-19, our data should be considered preliminary and should be confirmed in further studies. Disulfiram might be considered in patients with AUD and high risk of COVID-19 severe course. Future phase II/III randomized controlled trials should explore disulfiram efficacy on COVID-19 in the general population, since this drug may represent a cost-effective option for people with limited access to vaccines and specific antiviral drugs.

Acknowledgements Members of the Gruppo InterSERT di Collaborazione Scientifica (GICS) in alphabetical order: Costanza Agostoni, Roberta Almasio, Paola Avveduti, Ornella Baisini, Martina Ballerio, Alessandro Barbero, Marina Bellinato, Carlo Benevento, Loretta Bin, Veronica Bonizzi, Gianmario Borroni, Angelo Bove, Rita Broccardo, Sandra Bruschi, Roberta Brusini, Manuela Cairati, Liborio Martino Cammarata, Andrea Canale, Donatella Cantiero, Tania Capovilla, Maria Cristina Chiroli, Mauro Cibin, Raffaella Collesei, Daniela Colombo, Tiziana Cuomo, Annalisa D’Angelo, Susanna Dedola, Marco Degli Esposti, Giovanni De Micco, Vincenzo De Stefano, Filomena Di Cosmo, Tiziana Di Dio, Carmela Maria Di Fazio, Claudia Dona, Lorenza Fabiano, Mariacristina Fanton, Fulvio Fantozzi, Egle Maria Fergonzi, Elena Finotti, Francesca Fiore, Donatella Fiorentini, Nadia Fontana, Laura Franceschini, Luciana Furini, Paola Galletta, Francesco Gallo, Marianna Gardiolo, Roberta Gaspari, Nicola Gentile, Daniela Gervino, Novella Ghezzo, Cinzia Ghidoni, Maura Giubertoni, Paola Giussani, Filippo Gori, Francesca Groppa, Cinzia Grosina, Maria Raffaella Guzzetti, Paolo Jarre, Giovanna Maria Latino, Gianluca Lerro, Salvatore Lobello, Gloria Lobrano, Ilaria Valerida Lotti, Michael Malagò, Marcello Mazzo, Stefano Mellini, Cristina Meneguzzi, Rocco Mercuri, Sabrina Migliozzi, Elena Molinaroli, Antonio Mosti, Daniela Mussi, Alessandra Novelli, Anna Padovani, Carmela Palmigiano, Elio Panelli, Alessandro Pani, Serena Passaretti, Valentina Pavani, Maria Pecoraro, Ivo Peroglia, Marina Pitasi, Roberto Poli, Edoardo Polidori, Anna Laura Porcu, Davide Possetto, Antonio Prete, Serenella Quaresima, Paola Rapuzzi, Rosa Recchia, Fabio Reina, Marco Riglietta, Elisabetta Rizzo, Fabio Rugani, Maria Adele Salvadori, Stefania Samaia, Samantha Sanchini, Paolo Sandrone, Francesca Sassella, Antonia Scala, Michele Scarzella, Bruno Sciutteri, Ivana Sclaverano, Massimo Sorce, Elvira Speranza, Maria Caterina Staccioli, Laura Suardi, Fiorella Talassi, Anna Talluto, Biagio Tinghino, Sonia Tiso, Tania Tosi, Antonella Tusa, Maria Sabrina Ungari, Riccardo Valenti, Lucia Vecchi, Alessandro Vegliach, Rita Vercellone, Flavio Verdelli, Serena Vicario, Francesca Vignola, Cinzia Zambon, Angela Zannini, Ilenia Zanoni, Valeria Zavan, Cristina Zerbini.

Author contributions Study concept and design: ST, EB, DZ, FL. Acquisition of data: ST, EM, EB, DZ, FL. Analysis and interpretation of data: ST, EM, EB, DZ, FL. Drafting of the manuscript: ST, EM. Critical revision of the manuscript for important intellectual content: ST, EM, EB, DZ, FL. Statistical analysis: ST. Study supervision: FL. All the Authors read and approved the final version of the manuscript.

Funding This research did not receive any specific grant from funding agencies in the public, commercial, or not-for-profit sectors.
Availability of data and material The dataset generated for this study is available on request to the corresponding author.

\section{Compliance with ethical standards}

Conflict of interest No conflict declared.

Statements on human and animal rights The study was approved by the Verona University Hospital ethical committee (Comitato Etico per la Sperimentazione Clinica delle Province di Verona e Rovigo, Approval \# 2822CESC).

Consent to participate All the patients gave informed consent prior to the inclusion in the study.

\section{References}

1. Lin MH, Moses DC, Hsieh CH, Cheng SC, Chen YH, Sun CY, Chou CY (2018) Disulfiram can inhibit MERS and SARS coronavirus papain-like proteases via different modes. Antiviral Res 150:155-163

2. Li G, De Clercq E (2020) Therapeutic options for the 2019 novel coronavirus (2019-nCoV). Nat Rev Drug Discov 19:149-150

3. Boulware DR, Pullen MF, Bangdiwala AS, Pastick KA, Lofgren SM, Okafor EC, Skipper CP, Nascene AA, Nicol MR, Abassi M, Engen NW, Cheng MP, LaBar D, Lother SA, MacKenzie LJ, Drobot G, Marten N, Zarychanski R, Kelly LE, Schwartz IS, McDonald EG, Rajasingham R, Lee TC, Hullsiek KH (2020) A randomized trial of hydroxychloroquine as postexposure prophylaxis for Covid-19. N Engl J Med 3836:517-525

4. Italian Government Civil Protection Department. http://opend atadpc.maps.arcgis.com/apps/opsdashboard/index.html\#/b0c68 bce2cce478eaac82fe38d4138b1. Accessed 18 Jun 2020

5. Vollmer MAC, Mishra S, Unwin HJT, Gandy A, Mellan TA, Bradley V et al. (2020) Imperial College COVID-19 Response Team. Report 20: Using mobility to estimate the transmission intensity of COVID-19 in Italy: A subnational analysis with future scenarios. https://www.imperial.ac.uk/media/imperial-college/medic ine/mrc-gida/2020-05-04-COVID19-Report-20.pdf. Accessed 26 Oct 2020

6. Lenti MV, Borrelli de Andreis F, Pellegrino I, Klersy C, Merli S, Miceli E, Aronico N, Mengoli C, Di Stefano M, Cococcia S, Santacroce G, Soriano S, Melazzini F, Delliponti M, Baldanti F, Triarico A, Corazza GR, Pinzani M, Di Sabatino A, Internal Medicine Covid-19 Team (2020) Impact of COVID-19 on liver function: results from an internal medicine unit in Northern Italy. Intern Emerg Med 15:1399-1407

Publisher's Note Springer Nature remains neutral with regard to jurisdictional claims in published maps and institutional affiliations. 